\title{
Statistical Themes and Lessons for Data Mining
}

CLARK GLYMOUR

cg09@andrew.cmu.edu

Department of Cognitive Psychology, Carnegie Mellon University, Pittsburgh, PA 15213

DAVID MADIGAN

Department of Statistics, Box 354322, University of Washington, Seattle, WA 98195

madigan@stat.washington.edu

DARYL PREGIBON

Statistics Research, AT\&T Laboratories, Murray Hill, NJ 07974

PADHRAIC SMYTH

Information and Computer Science, University of California, Irvine, CA 92717

Editor: Usama Fayyad

Received June 27, 1996; Revised October 28, 1996; Accepted October 28, 1996 daryl@research.att.com

smyth@ics.uci.edu

\begin{abstract}
Data mining is on the interface of Computer Science and Statistics, utilizing advances in both disciplines to make progress in extracting information from large databases. It is an emerging field that has attracted much attention in a very short period of time. This article highlights some statistical themes and lessons that are directly relevant to data mining and attempts to identify opportunities where close cooperation between the statistical and computational communities might reasonably provide synergy for further progress in data analysis.
\end{abstract}

Keywords: statistics, uncertainty, modeling, bias, variance

\section{Introduction}

Sta-tis-tics (noun). The mathematics of the collection, organization, and interpretation of numerical data, especially the analysis of population characteristics by inference from sampling (American Heritage Dictionary).

Statistics is enjoying a renaissance period. Modern computing hardware and software have freed the statistician from narrowly specified models and spawned a fresh approach to the subject, especially as it relates to data analysis. Today's statistical toolkit draws on a rich body of theoretical and methodological research (Table 1).

The field of data mining, like statistics, concerns itself with "learning from data" or "turning data into information". The context encompasses statistics, but with a somewhat different emphasis. In particular, data mining involves retrospective analyses of data: thus, topics such as experimental design are outside the scope of data mining and fall within statistics proper. Data miners are often more interested in understandability than accuracy or predictability per se. Thus, there is a focus on relatively simple interpretable models 
Table 1. Statisticians have developed a large infrastructure (theory) to support their methods and a language (probability calculus) to describe their approach to quantifying the uncertainty associated with drawing inferences from data. These methods enable one to describe relationships between variables for prediction, quantifying effects, or suggesting causal paths.

\begin{tabular}{ll}
\hline Area of statistics & \multicolumn{1}{c}{ Description of activities } \\
\hline $\begin{array}{l}\text { Experimental design \& sampling } \\
\text { Exploratory data analysis }\end{array}$ & $\begin{array}{l}\text { How to select cases if one has the liberty to choose. } \\
\text { Hypothesis generation rather than hypothesis testing. } \\
\text { Statistical graphics }\end{array}$ \\
$\begin{array}{l}\text { Statistical modeling } \\
\text { Statistical inference }\end{array}$ & Regression and classification techniques. \\
\hline
\end{tabular}

involving rules, trees, graphs, and so forth. Applications involving very large numbers of variables and vast numbers of measurements are also common in data mining. Thus, computational efficiency and scalability are critically important, and issues of statistical consistency may be a secondary consideration. Furthermore, the current practice of data mining is often pattern-focused rather than model-focused, i.e., rather than building a coherent global model which includes all variables of interest, data mining algorithms (such as any of the many rule induction systems on the market) will produce sets of statements about local dependencies among variables (in rule form).

In this overall context, current data mining practice is very much driven by practical computational concerns. However, in focusing almost exclusively on computational issues, it is easy to forget that statistics is in fact a core component. The term "data mining" has long had negative connotations in the statistics literature (Selvin and Stuart, 1966; Chatfield, 1995). Data mining without proper consideration of the fundamental statistical nature of the inference problem is indeed to be avoided. However, a goal of this article is to convince the reader that modern statistics can offer significant constructive advice to the data miner, although many problems remain unsolved. Throughout the article we highlight some major themes of statistics research, focusing in particular on the practical lessons pertinent to data mining.

\section{An overview of statistical science}

This section briefly describes some of the central statistical ideas we think relevant to data mining. For a rigorous survey of statistics, the mathematically inclined reader should see, for example, Schervish (1995). For reasons of space we will ignore a number of interesting topics, including time series analysis and meta-analysis.

Probability distributions. The statistical literature contains mathematical characterizations of a wealth of probability distributions, as well as properties of random variablesfunctions defined on the "events" to which a probability measure assigns values. Important relations among probability distributions include marginalization (summing over a subset of values) and conditionalization (forming a conditional probability measure from a measure on a sample space and some event of positive measure). Essential relations among random variables include independence, conditional independence, and various measures 
of dependence, of which the most famous is the correlation coefficient. The statistical literature also characterizes families of distributions by properties that are useful in identifying any particular member of the family from data, or by closure properties useful in model construction or inference, for example conjugate families, closed under conditionalization, and the multinormal family, closed under linear combination. A knowledge of the properties of distribution families can be invaluable in analyzing data and making appropriate inferences.

Estimation, consistency, uncertainty, assumptions, robustness, and model averaging. An estimator is a function from sample data to some estimand, such as the value of a parameter. When the data comprise a sample from a larger actual or potential collection governed by some probability distribution, the family of estimators corresponding to all possible samples from that collection also has a probability distribution. Classical statistics investigates such distributions of estimators in order to establish basic properties such as reliability and uncertainty. A variety of resampling and simulation techniques also exist for assessing estimator uncertainty (Efron and Tibshirani, 1993).

Estimation almost always requires some set of assumptions. Such assumptions are typically false, but often useful. If a model (which we can think of as a set of assumptions) is incorrect, estimates based on it can be expected to be incorrect as well. One of the aims of statistical research is to find ways to weaken the assumptions necessary for good estimation. "Robust Statistics" (Huber, 1981) looks for estimators that work satisfactorily for larger families of distributions and have small errors when assumptions are violated.

Bayesian estimation emphasizes that alternative models and their competing assumptions are often plausible. Rather than making an estimate based on a single model, several models can be considered and an estimate obtained as the weighted average of the estimates given by the individual models (Madigan and Raftery, 1994). In fact, such Bayesian model averaging is bound to improve predictive performance, on average. Since the models obtained in data mining are usually the results of some automated search procedure, accounting for the potential errors associated with the search itself is crucial. In practice, this often requires a Monte Carlo analysis. Our impression is that the error rates of search procedures proposed and used in the data mining and in the statistical literature are far too rarely estimated in this way. (See Spirtes et al., 1993 for Monte Carlo test design for search procedures.)

Hypothesis testing. Since statistical tests are widely used, some of their important limitations should be noted. Viewed as a one-sided estimation method, hypothesis testing is inconsistent unless the alpha level of the testing rule is decreased appropriately as the sample size increases. Generally, an $\alpha$ level test of one hypothesis and an $\alpha$ level test of another hypothesis do not jointly provide an $\alpha$ level test of the conjunction of the two hypotheses. In special cases, rules (sometimes called contrasts) exist for simultaneously testing several hypotheses (Miller, 1981). An important corollary for data mining is that the $\alpha$ level of a test has nothing directly to do with the probability of error in a search procedure that involves testing a series of hypothesis. If, for example, for each pair of a set of variables, hypotheses of independence are tested at $\alpha=0.05$, then 0.05 is not the probability of erroneously finding some dependent set of variables when in fact all pairs are independent. Thus, in data mining procedures that use a sequence of hypothesis tests, the alpha level of the tests cannot generally be taken as an estimate of any error probability related to the outcome of the search. 
Data miners should note that while error probabilities of tests have something to do with the truth of hypotheses, the connection is somewhat tenuous (see Section 5.3). Hypotheses that are excellent approximations may be rejected in large samples; tests of linear models, for example, typically reject them in very large samples no matter how closely they seem to fit the data.

Model scoring. The evidence provided by data should lead us to prefer some models or hypotheses to others, and to be indifferent between still other models. A score is any rule that maps models and data to numbers whose numerical ordering corresponds to a preference ordering over the space of models, given the data. For the reasons just considered, scoring rules are often an attractive alternative to tests. Typical rules assign models a value determined by the likelihood function associated with the model, the number of parameters, or dimension, of the model, and the data. Popular rules include the Akaike Information Criterion (Akaike, 1974), Bayes Information Criterion (Raftery, 1995), and Minimum Description length (Rissanen, 1978). Given a prior probability distribution over models, the posterior probability on the data is itself a scoring function, arguably a privileged one. The Bayes Information Criterion approximates posterior probabilities in large samples.

There is a notion of consistency appropriate to scoring rules; in the large sample limit, almost surely the true model should be among those receiving maximal scores. AIC scores are not, in general, consistent (Schwartz, 1978). There are also uncertainties associated with scores, since two different samples of the same size from the same distribution may yield not only different numerical values for the same model, but even different orderings of models.

For obvious combinatorial reasons, it is often impossible when searching a large model space to calculate scores for all models; it is, however, often feasible to describe and calculate scores for a few equivalence classes of models receiving the highest scores.

In some contexts, inferences made using Bayesian scores can differ a great deal from inferences made with hypothesis tests. Raftery (1995) gives examples of models that account for almost all of the variance of an outcome of interest, and have very high Bayesian scores, but are overwhelmingly rejected by statistical tests.

Markov Chain Monte Carlo. Historically, insurmountable computational difficulties forced data analysts to eschew exact analysis of elaborate hierarchical Bayesian models and complex likelihood calculations. Recent dramatic advances in Monte Carlo methods have, however, liberated analysts from some of these constraints. One particular class of simulation methods, dubbed Markov Chain Monte Carlo, originally developed in statistical mechanics, has revolutionized the practice of Bayesian statistics. Smith and Roberts (1993) provide an accessible overview from the Bayesian perspective; Gilks et al. (1996) provide a practical introduction addressing both Bayesian and non-Bayesian perspectives.

Simulation methods may become unacceptably slow when faced with massive data sets. In such cases, recent advances in analytic approximations prove useful-see for example Kooperberg et al. (1996), Kass and Raftery (1995), and Geiger et al. (1996).

Generalized model classes. A major achievement of statistical methodological research has been the development of very general and flexible model classes. Generalized Linear 
Models, for instance, embrace many classical linear models, and unify estimation and testing theory for such models (McCullagh and Nelder, 1989). Generalized Additive Models show similar potential (Hastie and Tibshirani, 1990). Graphical models (Lauritzen, 1996) represent probabilistic and statistical models with planar graphs, where the vertices represent (possibly latent) random variables and the edges represent stochastic dependences. This provides a powerful language for describing models and the graphs themselves make modeling assumptions explicit. Graphical models provide important bridges between the vast statistical literature on multivariate analysis and such fields as artificial intelligence, causal analysis, and data mining.

Rational decision making and planning. The theory of rational choice assumes the decision maker has available a definite set of alternative actions, knowledge of a definite set of possible alternative states of the world, knowledge of the payoffs or utilities of the outcomes of each possible action in each possible state of the world, and knowledge of the probabilities of various possible states of the world. Given all of this information, a decision rule specifies which of the alternative actions ought to be taken. A large literature in statistics and economics addresses alternative decision rules-maximizing expected utility, minimizing maximum loss, etc. Typically, rational decision making and planning are the goals of data mining, and rather than providing techniques or methods for data mining, the theory of rational choice poses norms for the use of information obtained from a database.

The very framework of rational decision making requires probabilities and a knowledge of the effects alternative actions will have. To know the outcomes of actions is to know something of cause and effect relations, and extracting such causal information is often one of the principle goals of data mining and of statistical inference more generally.

Inference to causes. Understanding causation is the hidden force behind the historical development of statistics. From the beginning of the subject, in the work of Bernoulli and Laplace, the absence of causal connection between two variables has been taken to imply their probabilistic independence (see Stigler, 1986), and the same idea is fundamental in the theory of experimental design (Fisher, 1958). Early in this century, Wright (1921) introduced directed graphs to represent causal hypotheses (with vertices as random variables and edges representing direct influences), and they have become common representations of causal hypotheses in the social sciences, biology, computer science and engineering.

Kiiveri and Speed (1982) combined directed graphs with a generalized connection between independence and absence of causal connection in what they called the Markov condition: provided $Y$ is not an effect of $X, X$ and $Y$ are conditionally independent given the direct causes of $X$. They showed that much of the linear modeling literature tacitly assumed the Markov condition; the same is true for causal models of categorical data, and virtually all causal models of systems without feedback. Under additional assumptions, conditional independence therefore provides information about causal dependence. The most common, and most thoroughly investigated, additional assumption is that all conditional independencies are due to the Markov condition applied to the directed graph describing the actual causal processes generating the data, a requirement that has been given many names, including "faithfulness". Directed graphs with associated probability distributions satisfying the Markov condition are called by different names in different literatures: Bayes nets, 
belief nets, structural equation models, path models, etc. Nonetheless, causal inferences from uncontrolled convenience samples are liable to many sources of error and data miners should proceed with extreme caution.

Sources of error peculiar to causal inference from a database include the existence of unrecorded causes of associations (confounders) between recorded variables, associations among recorded variables produced by the influence such variables themselves have on whether a unit is included in the database or the sample drawn from it (sample selection bias), missing data (which can be produced either by confounding or by sample selection bias), and samples composed of units with different causal structures. Assuming that all independencies result from the Markov property of directed graphs representing causal hypotheses, for multinormal and multinomial distributions there are procedures based on hypothesis tests of constraints that, in the large sample limit, give correct causal information under most of these conditions (Scheines, 1994; Spirtes, et al. 1995). Scoring search algorithms have been developed for the case where there are no confounders, no mixtures and no sample selection bias, but the best performance in search seems to be obtained using hypothesis-test initial searches followed by a Bayes score post search (Spirtes and Meek, 1995). Work is under way developing feasible scoring searches for latent variable models (Geiger, 1996; Spirtes, 1997).

Prediction. Sometimes one is interested in using a sample, or a database, to predict properties of a new sample, where it is assumed that the two samples are obtained from the same probability distribution. As with estimation, in prediction we are interested both in reliability and in uncertainty, often measured by the variance of the predictor.

Prediction methods for this sort of problem always assume some structure in the probability distribution. In data mining contexts, structure is typically either supplied by human experts, or inferred from the database automatically. Regression, for example, assumes a particular functional form relating variables. Structure can be also be specified in terms of constraints, such as independence, conditional independence, higher order conditions on correlations, etc. On average, a prediction method that guarantees satisfaction of the constraints realized in the probability distribution-and no others-will be more accurate and have smaller variance than one that does not. Finding the appropriate constraints to satisfy is the most difficult issue in this sort of prediction. As with estimation, prediction can be improved by model averaging, provided the prior probabilities of the alternative assumptions imposed by the model are available.

Often the results of data mining are used to attempt to predict the effects of a policy change or intervention of some kind. Such predictions are essentially causal inferences, and are not in general correctly given by estimating a probability distribution and predicting by conditioning on the variable to be manipulated.

\section{Is data mining "statistical deja vu” (all over again)?}

In the mid 1960's, the statistics community referred to unfettered exploration of data as "fishing" or "data dredging" (Selvin and Stuart, 1966). The community, enamored by elegant (analytical) mathematical solutions to inferential problems, argued that since their 
theories were invalidated by "looking at the data", it was wrong to do so. The major proponent of the exploratory data analysis (EDA) school, J.W. Tukey, countered this argument with the obvious retort that statisticians were putting the cart before the horse. He argued that statistical theory should adapt to the scientific method rather than the other way around. Thirty years hence, the statistical community has largely adopted Tukey's perspective, and has made considerable progress in serving both masters, namely acknowledging that model search is a critical and unavoidable step in the modeling process, and devising formal methods to account for search in their inferential procedures.

Three themes of modern statistics that are of fundamental importance to data miners are: clarity about goals, appropriate reliability assessment, and adequate accounting for sources of uncertainty.

Clarity about goals. Sometimes data analysis aims to find a convenient, easily computable representation of how the data are distributed in a particular database. In other cases, data analysis aims to predict features of new cases, or new samples, drawn from outside the database used to develop a predictive model (this is particularly challenging in dynamic situations). In yet other cases, data analysis aims to provide a basis for policy. That is, the analysis is intended to yield insight into causal mechanisms that are used to form predictions about new samples that might be produced by interventions or actions that did not apply in the original database from which the model (or models) were developed. Each of these goals present distinct inference problems, with distinct hazards. Confusing or equivocating over the aim invites the use of inappropriate methods and may result in unfortunate predictions and inferences.

As an example, consider the observational study reported by Chasnoff et al. (1989) comparing babies born to cocaine-using mothers with babies born to non-cocaine-using mothers. The authors concluded: "For women who become pregnant and are users of cocaine, intervention in early pregnancy with cessation of cocaine use will result in improved obstetric outcome". Fortunately, there exists independent evidence to support this causal claim. However, much of Chasnoff et al.'s paper focuses on a statistical analysis (analysis of variance) that has little, if anything, to do with the causal question of interest.

Hand (1994) provides a series of examples illustrating how easy it is to give the right answers to the wrong question. For example, he discusses the problem of analyzing clinical trial data where patients drop out due to adverse side-effects of a particular treatment (Diggle and Kenward, 1994). In this case, the important issue is which population is one interested in modelling? The population at large versus the population who remain within the trial? This problem arises in more general settings than in clinical trials, e.g., non-respondents (refusers) in survey data. In such situations it is important to be explicit about the questions one is trying to answer.

In this general context an important issue (discussed at length in Hand (1994)) is that of formulating statistical strategy i.e., how does one structure a data analysis problem so that the right question can be asked? Hand's conclusion is that this is largely an "art" because it is less well formalized than the mathematical and computational details of applying a particular technique. This "art" is gained through experience (at present at least) rather than taught. The implication for data mining is that human judgment is essential for many non-trivial inference problems. Thus, automation can at best only partially guide the data 
analysis process. Properly defining the goals of an analysis remains a human-centred, and often difficult, process.

Use of methods that are reliable means to the goal, under assumptions the user (and consumer) understands and finds plausible in the context. Statistical theory applies several meanings to the word "Reliability", many of which also apply to model search. For example, under what conditions does a search procedure provide correct information, of the kind sought, with probability one as the sample size increases without bound? Answers to such questions are often elusive and can require sophisticated mathematical analysis. Where answers are available, the data analyst should pay careful attention to the reasonableness of underlying assumptions. Another key data mining question is this: what are the probabilities of various kinds of errors that result from using a method in finite samples? The answers to this question will typically vary with the kinds of errors considered, with the sample size, and with the frequency of occurrence of the various kinds of targets or signals whose description is the goal of inference. These questions are often best addressed by Monte Carlo methods, although in some cases analytic results may be available.

A sense of the uncertainties of models and predictions. Quite often background knowledge and even the best methods of search and statistical assessment should leave the investigator with a range of uncertainties about the correct model, or the correct prediction. The data analyst must quantify these uncertainties so that subsequent decisions can be appropriately hedged. Section 4 provides a compelling example.

Another example involves a current debate in the atmospheric sciences. The question is whether or not specific recurrent pressure patterns can be clearly identified from daily geopotential height records which have been compiled in the Northern Hemisphere since 1948. The existence of well-defined recurrent patterns (or "regimes") has significant implications for models of upper atmosphere low-frequency variability beyond the time-scale of daily weather disturbances (and, thus, models of the earth's climate over large time-scales). Several studies have used a variety of clustering algorithms to detect inhomogeneities ("bumps") in low-dimensional projections of the gridded data (see Michelangeli et al. (1995) and others referred to therein). While this work has attempted to validate the cluster models via resampling techniques, it is difficult to infer from the multiple studies whether regimes truly exist, and, if they do, where precisely they are located. It seems likely that 48 winters worth of data is not enough to identify regimes to any degree of certainty and that there is a fundamental uncertainty (given the current data) about the underlying mechanisms at work. All is not lost, however, since it is also clear that one could quantify model uncertainty in this context, and theorize accordingly (see Section 4).

In what follows we will elaborate on these points and offer a perspective on some of the hazards of data mining.

\section{Characterizing uncertainty}

The statistical approach contends that reporting a single number for a parameter estimate or a prediction is almost always inadequate. Quantification of the uncertainty associated 
with a single number, while often challenging, is critical for subsequent decision making. As an example, Draper (1995), considered the case of the 1980 Energy Modeling Forum (EMF) at Stanford University where a 43-person working group of economists and energy experts convened to forecast world oil prices from 1981 to 2020. The group generated predictions based on a number of econometric models and scenarios, embodying a variety of assumptions about supply, demand, and growth rates of relevant quantities. A plausible reference scenario and model was selected as representative, but the summary report (EMF, 1982) cautioned against interpreting point predictions based on the reference scenario as "[the working group's] 'forecast' of the oil future, as there are too many unknowns to accept any projection as a forecast". The summary report did conclude, however, that most of the uncertainty about future oil prices "concerns not whether these prices will rise ... but how rapidly they will rise".

In 1980, the average spot price of crude oil was around $\$ 32$ per barrel. Despite the warning about the potential uncertainty associated with the point estimates, governments and private companies around the world focused on the last sentence in the quotation above, and proceeded to invest an estimated $\$ 500$ billion dollars, on the basis that the price would probably be close to $\$ 40$ dollars per barrel in the mid-eighties. In fact, the actual 1986 world average spot price of oil was about $\$ 13$ per barrel.

Using only the information available to the EMF in 1980, along with thoughtful but elementary statistical methods, Draper (1995) shows that a $90 \%$ predictive interval for the 1986 price would have ranged from about $\$ 20$ to over $\$ 90$. Note that this interval does not actually contain the actual 1986 price-insightful statistical analysis does not provide clairvoyance. However, decision makers would (and should) have proceeded more cautiously in 1980, had they understood the full extent of their uncertainty.

Correctly accounting for the different sources of uncertainty presents significant challenges. Until recently, the statistical literature focused primarily on quantifying parametric and predictive uncertainty in the context of a particular model. Two distinct approaches are in common use. "Frequentist" statisticians focus on the randomness in sampled data and summarize the induced randomness in parameters and predictions by so-called sampling distributions. "Bayesian" statisticians instead treat the data as fixed, and use Bayes Theorem to turn prior opinion about quantities of interest (always expressed by a probability distribution), into a so-called posterior distribution that embraces all the available information. The fierce conflicts between previous generations of frequentists and Bayesians, have largely given way in recent years to a more pragmatic approach; most statisticians will base their choice of tool on scientific appropriateness and convenience.

In any event, recent research has lead to increased awareness that within-model uncertainty (as discussed in the previous paragraph) may often, in practice, be dominated by between-model uncertainty (Chatfield, 1995, Draper, 1995, Madigan and York, 1995). It is common practice nowadays for statisticians and data miners to use computationally intensive model selection algorithms to seek out a single optimal model from an enormous class of potential models. The problem is that several different models may be close to optimal, yet lead to different inferences. Intuitively, ambiguity over the model should dilute information about effect parameters and predictions, since "part of the evidence is spent to specify the model" (Leamer, 1978, p. 91). Promising techniques for properly accounting for 
this source of uncertainty include Bayesian model averaging (Draper, 1995) and resampling methods (Breiman, 1996). The main point here is that data miners need to think carefully about model assessment and look beyond commonly used goodness-of-fit measures such as mean square error.

\section{What can go wrong, will go wrong}

Data mining poses difficult and fundamental challenges to the theory and practice of statistics. While statistics does not have all the answers for the data miner, it does provide a useful and practical framework for which to search for solutions. In this Section, we describe some lessons that statisticians have learned when theory meets data.

\subsection{Data can lie}

Data mining applications typically rely on observational (as opposed to experimental) data. Interpreting observed associations in such data is challenging; sensible inferences require careful analysis, and detailed consideration of the underlying factors. Here we offer a detailed example to support this position.

Wen et al. (1995; WHN hereafter) analyzed administrative records of all Ontario general hospital separations (discharges, transfers, or in-hospital deaths) from 1981 to 1990, focusing specifically on patients who had received a primary open cholecystectomy. Some of these patients had in addition received an incidental (i.e., discretionary) appendectomy during the cholecystectomy procedure. Table 2 displays the data on one outcome, namely in-hospital deaths. A chi-square test comparing this outcome for the two groups of patients shows a "statistically significant" difference. This "finding" is surprising since long-term prevention of appendicitis is the sole rationale for the incidental appendectomy procedureno short-term improvement in outcomes is expected. This "finding" might lead a naive hospital policy maker to conclude that all cholecystectomy patients should have an incidental appendectomy to improve their chances of a good outcome! Clearly something is amiss - how could incidental appendectomy improve outcomes?

WHN did separately consider a subgroup of low-risk patients. For these patients (using ten different definitions of "low-risk"), incidental appendectomy indeed resulted in poorer outcomes. Paradoxically, it could even be the case that appendectomy adversely affects outcomes for both high-risk patients and low-risk patients, but appears to positively affect outcomes when the low-risk and high-risk patients are combined. WHN do not provide enough data to check whether this so-called "Simpson's Paradox" (Simpson, 1951)

Table 2. In-hospital survival of patients undergoing primary open cholecystectomy with and without incidental appendectomy.

\begin{tabular}{lcc}
\hline & $\begin{array}{c}\text { With } \\
\text { appendectomy }\end{array}$ & $\begin{array}{c}\text { Without } \\
\text { appendectomy }\end{array}$ \\
\hline In-hospital deaths, No. (\%) & $21(0.27 \%)$ & $1,394(0.73 \%)$ \\
In-hospital survivors, No. (\%) & $7,825(99.73 \%)$ & $190,205(99.27 \%)$ \\
\hline
\end{tabular}


Table 3. Fictitious data consistent with the Wen et al. (1995) data.

\begin{tabular}{lccrrr}
\hline & \multicolumn{2}{c}{ With appendectomy } & & \multicolumn{2}{c}{ Without appendectomy } \\
\cline { 2 - 3 } \cline { 5 - 6 } & Low-risk & High-risk & & Low-risk & High-risk \\
\hline Death & 7 & 14 & & 100 & 1294 \\
Survival & 7700 & 125 & & 164009 & 26196 \\
\hline
\end{tabular}

Table 4. Proportion of in-hospital deaths cross-classified by incidental appendectomy and patient risk grouping for the fictitious data of Table 3 .

\begin{tabular}{lcc}
\hline & $\begin{array}{c}\text { With } \\
\text { appendectomy }\end{array}$ & $\begin{array}{c}\text { Without } \\
\text { appendectomy }\end{array}$ \\
\hline Low-risk & 0.0009 & 0.0006 \\
High-risk & 0.10 & 0.05 \\
Combined & 0.003 & 0.007 \\
\hline
\end{tabular}

occurred in this example. However, Table 3 presents data that are plausible and consistent with WHN's data.

Table 4 displays the corresponding proportions of in-hospital death for these fictitious data. Clearly the risk and death categories are directly correlated. In addition, appendectomies are more likely to be carried out on low-risk patients than on high-risk ones. Thus, if we did not know the risk category (age) of a patient, knowing that they had an appendectomy allows us to infer that they are more likely to be lower risk (younger). However, this does not in any way imply that having an appendectomy will lower one's risk. Nonetheless, when risk is omitted from the table, exactly such a fallacious conclusion appears justified from the data.

Returning to the original data, WHN provide a more sophisticated regression analysis, adjusting for many possible confounding variables (e.g., age, sex, admission status). They conclude that "there is absolutely no basis for any short-term improvement in outcomes" due to incidental appendectomy. This careful analysis agrees with common sense in this case. In general, analyses of observational data demand such care, and come with no guarantees. Other characteristics of available data that connive to spoil causal inferences include:

- Associations in the database may be due in whole or part to unrecorded common causes (latent variables).

- The population under study may be a mixture of distinct causal systems, resulting in statistical associations that are due to the mixing rather than to any direct influence of variables on one another or any substantive common cause.

- Missing values of variables for some units may result in misleading associations among the recorded values.

- Membership in the database may be influenced by two or more factors under study, which will create a "spurious" statistical association between those variables. 
- Many models with quite distinct causal implications may "fit" the data equally or almost equally well.

- The frequency distributions in samples may not be well approximated by the most familiar families of probability distributions.

- The recorded values of variables may be the result of "feedback" mechanisms which are not well represented by simple "non-recursive" statistical models.

There is research that addresses aspects of these problems, but there are few statistical procedures yet available that can be used "off the shelf"- - the way randomization is used in experimental design - to reduce these risks. Standard techniques such as multiple regression, and logistic regression may work in many cases, such as in the appendectomy example, but they are not always adequate guards against these hazards. Indeed, controlling for possibly confounding variables with multiple regression can in some cases produce inferior estimates of effect sizes. Procedures recently developed in the artificial intelligence and statistics literature (Spirtes et al., 1993) address some of the problems associated with latent variables and mixing, but so far only for two families of probability distributions, the normal and multinomial.

\subsection{Sometimes it's not what's in the data that matters}

Classical statistical methods start with a random sample, yet in practice, data or the institutions that give rise to data, can be uncooperative. In such cases, inferences that ignore how the data were "selected" can lead to distorted conclusions.

Consider, for example, the Challenger Space Shuttle accident. The Rogers Commission concluded that an O-ring failure in the solid rocket booster led to the structural breakup and loss of the Challenger. In reconstructing the events leading up to the decision to launch, the Commission noted a mistake in the analysis of thermal-distress data whereby flights with no (i.e., zero) incidents of O-ring damage were excluded from critical plots of O-ring damage and ambient launch temperature since it was felt that they did not contribute any information about the temperature effect. This truncation of the data led to the conclusion that no relationship between O-ring damage and temperature existed, and ultimately, the decision to launch. Dalal et al. (1989) throw statistical light on the matter by demonstrating the strong correlation between O-ring damage and temperature, and quantifying the risk (of catastrophic failure) at $31^{\circ} \mathrm{F}$. Had the original analysis used all of the data, it would have indicated that the decision to launch was at best a risky proposition.

In the above case, the selection bias problem was one of "human error" and could easily have been avoided. In most problems, selection bias is an inherent characteristic of the available data and methods of analysis need to deal with it. It is our experience that every data set has the potential for selection bias to invalidate standard inferences. The lessons to be learned here are

- that any technique used to analyze truncated data as if it was a random sample, can be fooled, regardless of how the truncation was induced;

- the data themselves are seldom capable to alert the analyst that a selection mechanism is operating - information external to the data at hand is critical in understanding the nature and extent of potential biases. 


\subsection{The perversity of the pervasive P-value}

$P$-values and associated significance (or hypothesis) tests play a central role in classical (frequentist) statistics. It seems natural, therefore, that data miners should make widespread use of $P$-values. However, indiscriminate use of $P$-values can lead data miners astray in most applications.

The standard significance test proceeds as follows. Consider two competing hypotheses about the world: the Null Hypothesis, commonly denoted by $H_{0}$, and the Alternative Hypothesis, commonly denoted by $H_{A}$. Typically $H_{0}$ is "nested" within $H_{A}$; for example, $H_{0}$ might state that a certain combination of parameters is equal to zero, while $H_{A}$ might place no restriction on the combination. A test statistic, $T$ is selected and calculated from the data at hand. The idea is that $T$ (Data) should measure the evidence in the data against $H_{0}$. The analyst rejects $H_{0}$ in favor of $H_{A}$ if $T$ (Data) is more extreme than would be expected if $H_{0}$ were true. Specifically, the analyst computes the $P$-value, that is, the probability of $T$ being greater than or equal to $T$ (Data), given that $H_{0}$ is true. The analyst rejects $H_{0}$ if the $P$-value is less than a preset significance level, $\alpha$.

There are three primary difficulties associated with this approach:

1. The standard advice that statistics educators provide, and scientific journals rigidly adhere to, is to choose $\alpha$ to be 0.05 or 0.01 , regardless of sample size. These particular $\alpha$-levels arose in Sir Ronald Fisher's study of relatively small agricultural experiments (on the order of 30-200 plots). Textbook advice (e.g., Neyman and Pearson, 1933) has emphasized the need to take account of the power of the test against $H_{A}$ when setting $\alpha$, and somehow reduce $\alpha$ when the sample size is large. This crucial but vague advice has largely fallen on deaf ears.

2. Raftery (1995) points out that the whole hypothesis testing framework rests on the basic assumption that only two hypotheses are ever entertained. In practice, data miners will consider very large numbers of possible models. As a consequence, indiscriminate use of $P$-values with "standard" fixed $\alpha$-levels can lead to undesirable outcomes such as selecting a model with parameters that are highly significantly different from zero, even when the training data are pure noise (Freedman, 1983). This point is of fundamental importance for data miners.

3 . The $P$-value is the probability associated with the event that the test statistic was as extreme as the value observed, or more so. However, the event that actually happened was that a specific value of the test statistic was observed. Consequently, the relationship between the $P$-value and the veracity of $H_{0}$ is subtle at best. Jeffreys (1980) puts it this way:

I have always considered the arguments for the use of $P$ absurd. They amount to saying that a hypothesis that may or may not be true is rejected because a greater departure from the trial value was improbable; that is, that it has not predicted something that has not happened.

Bayes Factors are the Bayesian analogue of the frequentist $P$-values and admit to a more direct interpretation-the Bayesian analyst computes the posterior probability that a hypothesis is correct. With fixed $\alpha$-levels, the frequentist and the Bayesian will arrive at very different conclusions. For example, Berger and Sellke (1987) show that data that yield 
a $P$-value of 0.05 when testing a normal mean, result in a posterior probability for $H_{0}$ that is at least 0.30 for any "objective" prior distribution. One way to reconcile the two positions is to view Bayes Factors as a method for selecting appropriate $\alpha$-levels—see Raftery (1995).

\subsection{Intervention and prediction}

A specific class of prediction problems involve interventions that alter the probability distribution of the problem, as in predicting the values (or probabilities) of variables under a change in manufacturing procedures, or changes in economic or medical treatment policies. Accurate predictions of this kind require some knowledge of the relevant causal structure, and are in general quite different from prediction without intervention, although the usual caveats about uncertainty and model averaging apply. For graphical representations of causal hypotheses according to the Markov condition, general algorithms for predicting the outcomes of interventions from complete or incomplete causal models were developed in Spirtes et al. (1993). Some of these procedures have been extended and made into a more convenient calculus by Pearl (1995). A related theory without graphical models was developed earlier by Rubin (1974) and others, and by Robbins (1986).

Consider the following example. Herbert Needleman's famous studies of the correlation of lead deposits in children's teeth with their IQs resulted, eventually, in removal of tertraethyl lead from gasoline in the United States. One data set Needleman examined included more than 200 subjects, and measured a large number of covariates. Needleman et al. (1985) re-analyzed the data using backwards step-wise regression of verbal IQ on these variables and obtained six significant regressors, including lead. Klepper (1988) reanalyzed the data assuming that all of the variables were measured with error. Their model assumes that each measured number is a linear combination of the true value and an error, and that the parameters of interest are not the regression coefficients but rather the coefficients relating the unmeasured "true value" variables to the unmeasured true value of verbal IQ. These coefficients are in fact indeterminate-in econometric terminology, "unidentifiable". An interval estimate of the coefficients that is strictly positive or negative for each coefficient can be made, however, if the amount of measurement error can be bounded with prior knowledge by an amount that varies from case to case. Klepper found that the bound required to ensure the existence of a strictly negative interval estimate for the lead-IQ coefficient was much too strict to be credible, thus he concluded that the case against lead was not nearly as strong as Needleman's analysis suggested.

Allowing the possibility of latent variables, Scheines (1996) reanalyzed the correlations (using TETRAD methodology) and concluded that three of the six regressors could have no influence on IQ. The regression included the three extra variables only because the partial regression coefficient is estimated by conditioning on all other regressors, which is just the right thing to do for linear prediction, but the wrong thing to do for causal inference using the Markov condition. Using the Klepper model, but without the three irrelevant variables, and assigning to all of the parameters a normal prior probability with mean zero and a substantial variance, Scheines then used Markov Chain Monte Carlo to compute a posterior probability distribution for the lead-IQ parameter. The probability is very high that lead exposure reduces verbal IQ. 


\section{Symbiosis in statistics}

Easy access to data in digital form and the availability of software tools for statistical analyses have made it possible for the man in street to set up shop and "do statistics". Nowhere is this more true today than in data mining. Based on the arguments in this article, let us assume that statistics is a necessary but not sufficient component in the practice of data mining. How well will the statistics profession serve the data mining community? Hoerl et al. (1993), for example, assert that:

We are our own best customers. Much of the work of the statistical profession is intended for other members of the statistical profession.

Despite this rather negative view of the relevance of statistical research, real-world applications do in fact drive much of what goes on it statistics, although often in a very indirect manner.

As an example consider the field of signal processing and communications, an area where a specialized set of relatively sophisticated statistical methods and models have been honed for practical use. The field was driven by fundamental advances from Claude Shannon and others in the 1940's. Like most of the other contributors to the field, Shannon was not a statistician, but possessed a deep understanding of probability theory and its applications. Through the 1950's to the present, due to rapid advances in both theory and hardware, the field has exploded and relevant statistical methods such as estimation and detection have found their way into everyday use in radio and network communications systems. Modern statistical communications reflects the symbiosis of statistical theory and engineering practice. Engineering researchers in the field are in effect "adjunct" statisticians: educated in probability theory and basic statistics they have the tools to apply statistical methods to their problems of interest. Meanwhile statisticians continue to develop more general models and estimation techniques of potential applicability to new problems in communications.

This type of symbiosis can also be seen in other areas such as financial modelling, speech recognition (where for example hidden Markov models provide the state-of-the-art in the field), and most notably, epidemiology. Indeed, if statistics can claim to have revolutionized any field, it is in the biological and health sciences where the statistical approach to data analysis gave birth to the field of biostatistics.

The relevance of this symbiosis for data mining is that data-miners need to understand statistical principles, and statisticians need to understand the nature of the important problems that the data mining community is attacking or being asked to attack. This has been a successful model in the past for fields where statistics has had considerable impact and has the potential to see ongoing success.

\section{Conclusion}

The statistical literature has a wealth of technical procedures and results to offer data mining, but it also has a few simple methodological morals: prove that estimation and search procedures used in data mining are consistent under conditions reasonably thought to apply in applications; use and reveal uncertainty, don't hide it; calibrate the errors of search, both 
for honesty and to take advantages of model averaging; don't confuse conditioning with intervening; and finally, don't take the error probabilities of hypothesis tests to be the error probabilities of search procedures.

\section{References}

Akaike, H. 1974. A new look at the statistical model identification. IEEE Trans. Automat. Contr., AC-19:716-723.

Berger, J.O. and Sellke, T. 1987. Testing a point null hypothesis: The irreconcilability of $P$ values and evidence (with discussion). Journal of the American Statistical Association, 82:112-122.

Breiman, L. 1996. Bagging predictors. Machine Learning (to appear).

Chasnoff, I.J., Griffith, D.R., MacGregor, S., Dirkes, K., and Burns, K.A. 1989. Temporal patterns of cocaine use in pregnancy: Perinatal outcome. Journal of the American Medical Association, 261(12):1741-1744.

Chatfield, C. 1995. Model uncertainty, data mining, and statistical inference (with discussion). Journal of the Royal Statistical Society (Series A), 158:419-466.

Dalal, S.R., Fowlkes, E.B., and Hoadley, B. 1989. Risk analysis of the space shuttle: Pre-Challenger prediction of failure. Journal of the American Statistical Association, 84:945-957.

Diggle, P. and Kenward, M.G. 1994. Informative drop-out in longitudinal data analysis (with discussion). Applied Statistics, 43:49-93.

Draper, D. 1995. Assessment and propagation of model uncertainty (with discussion). Journal of the Royal Statistical Society (Series B), 57:45-97.

Draper, D., Gaver, D.P., Goel, P.K., Greenhouse, J.B., Hedges, L.V., Morris, C.N., Tucker, J., and Waternaux, C. 1993. Combining information: National Research Council Panel on Statistical Issues and Opportunities for Research in the Combination of Information. Washington: National Academy Press.

Efron, B. and Tibshirani, R.J. 1993. An Introduction to the Boostrap. New York: Chapman and Hall.

Energy Modeling Forum. World Oil: Summary report. EMF Report 6, Energy Modeling Forum. Stanford University, Stanford, CA, 1982.

Fisher, R.A. 1958. Statistical methods for research workers. New York: Hafner Pub. Co.

Freedman, D.A. 1983. A note on screening regression equations. The American Statistician, 37:152-155.

Geiger, D., Heckerman, D., and Meek, C. 1996. Asymptotic model selection for directed networks with hidden variables. Proceedings of the Twelfth Annual Conference on Uncertainty in Artificial Intelligence. San Francisco: Morgan Kaufman.

Gilks, W.R., Richardson, S., and Spiegelhalter, D.J. 1996. Markov Chain Monte Carlo in practice. London: Chapman and Hall.

Hand, D.J. 1994. Deconstructing statistical questions (with discussion). Journal of the Royal Statistical Society (Series A), 157:317-356, 1994.

Hastie, T.J. and Tibshirani, R. 1990. Generalized Additive Models. London: Chapman and Hall.

Hoerl, R.W., Hooper, J.H., Jacobs, P.J., and Lucas, J.M. 1993. Skills for industrial statisticians to survive and prosper in the emerging quality environment. The American Statistician, 47:280-292.

Huber, P.J. 1981. Robust Statistics. New York: Wiley.

Jeffreys, H. 1980. Some general points in probability theory. In Bayesian Analysis in Econometrics and Statistics, A. Zellner (Ed.). Amsterdam: North-Holland, 451-454.

Kass, R.E. and Raftery, A.E. 1995. Bayes factors. Journal of the American Statistical Association, 90:773-795.

Kiiveri, H. and Speed, T.P. 1982. Structural analysis of multivariate data: A review. Sociological Methodology, 209-289.

Kooperberg, C., Bose, S., and Stone, C.J. 1996. Polychotomous regression. Journal of the American Statistical Association (to appear).

Lauritzen, S.L. 1996. Graphical Models. Oxford: Oxford University Press.

Leamer, E.E. 1978. Specification Searches. Ad Hoc Inference with Nonexperimental Data. New York: Wiley.

Madigan, D. and Raftery, A.E. 1994. Model selection and accounting for model uncertainty in graphical models using Occam's Window. Journal of the American Statistical Association, 89:1335-1346.

Madigan, D. and York, J. 1995. Bayesian graphical models for discrete data. International Statistical Review, $63: 215-232$. 
Matheson, J.E. and Winkler, R.L. 1976. Scoring rules for continuous probability distributions. Management Science, 22:1087-1096.

McCullagh, P. and Nelder, J.A. 1989. Generalized Linear Models. London: Chapman and Hall.

Michelangeli, P.A., Vautard, R., and Legras, B. 1995. Weather regimes: recurrence and quasi-stationarity. Journal of the Atmospheric Sciences, 52(8):1237-1256.

Miller, R.G., Jr. 1981. Simultaneous statistical inference (second edition). New York: Springer-Verlag.

Neyman, J. and Pearson, E.S. 1933. On the problem of the most efficient tests of statistical hypotheses. Philosophical Transactions of the Royal Society (Series A), 231:289-337.

Pearl, J. and Verma, T. 1990. A formal theory of inductive causation. Technical Report R-155, Cognitive Systems Laboratory, Computer Science Dept. UCLA.

Pearl, J. and Verma, T. 1991. A theory of inferred causation. Principles of knowledge representation and reasoning. Proceedings of the Second International Conference, Morgan Kaufmann, San Mateo, CA.

Pearl, J. 1995. Causal diagrams for empirical research. Biometrika. 82(4):669-709.

Raftery, A.E. 1995. Bayesian model selection in social research (with discussion). In Sociological Methodology, P.V. Marsden (Ed.). Oxford, U.K.: Blackwells, pp. 111-196.

Rissanen, J. 1978. Modeling by shortest data description. Automatica, 14:465-471.

Scheines, R., Spirtes, P., Glymour, C., and Meek, C. 1994. TETRAD II: Users Manual, Lawrence Erlbaum Associates, Hillsdale, NJ.

Scheines, R., Hoijtink, H., and Boomsma, A. 1995. Bayesian estimation and testing of structural equation models. Technical Report CMU-PHIL-66, Dept. of Philosophy, Carnegie Mellon Univ., Pgh, PA, 15213.

Schervish, M.J. 1995. Theory of Statistics, New York: Springer Verlag.

Schwartz, G. 1978. Estimating the dimension of a model. Annals of Statistics, 6:461-464.

Selvin, H. and Stuart, A. 1966. Data dredging procedures in survey analysis. The American Statistician, 20(3):2023.

Simpson, C.H. 1951. The interpretation of interaction in contingency tables. Journal of the Royal Statistical Society (Series B), 13:238-241.

Smith, A.F.M. and Roberts, G. 1993. Bayesian computation via the Gibbs sampler and related Markov Chain Monte Carlo methods (with discussion). Journal of the Royal Statistical Society (Series B), 55:3-23.

Spirtes, P., Glymour, C., and Scheines, R. 1993. Causation, Prediction and Search. Springer Lecture Notes in Statistics. New York: Springer Verlag.

Spirtes, P. and Meek, C. 1995. Learning Bayesian networks with discrete variables from data. Proceeding of the First International Conference on Knowledge Discovery and Data Mining, Usama M. Fayyad and Ramasamy Uthurusamy (Eds.), AAI Press, pp. 294-299.

Spirtes, P., Meek, C., and Richardson, T. 1995. Causal inference in the presence of latent variables and selection bias. Proceedings of the Eleventh Conference on Uncertainty in Artificial Intelligence, Philippe Besnard and Steve Hanks (Eds.), Morgan Kaufmann Publishers, Inc., San Mateo, CA, pp. 499-506.

Spirtes, P. 1997. Heuristic greedy search algorithms for latent variable models. Proceedings of the Conference on $\mathrm{AI}$ and Statistics, Fort Lauderdale, forthcoming.

Stigler, S.M. 1986. The history of statistics: The measurement of uncertainty before 1900. Harvard: Harvard University Press.

Wen, S.W., Hernandez, R., and Naylor, C.D. 1995. Pitfalls in nonrandomized studies: The case of incidental appendectomy with open cholecystectomy. Journal of the American Medical Association, 274:1687-1691.

Wright, S. 1921. Correlation and causation. Journal of Agricultural Research, 20:557-585.

Clark Glymour is Valtz Family Professor of Philosophy at the University of California, San Diego, Alumni University Professor at Carnegie Mellon University, and this year, with support from the Andrew Mellon foundation, a fellow of the Center For Advanced Study in the Behavioral Sciences. He is the co-author of Causation, Prediction and Search (Springer, 1993), and Discovering Causal Structure (1987), and the author of Thinking Things Through (MIT, 1993). His present interests are in applications of artificial intelligence in applied statistics, and in applications of computational learning theory in psychology.

David Madigan is an Associate Professor of Statistics at the University of Washington. He received a PhD degree from Trinity College Dublin in 1990. He has research interests in technology-assisted learning, Bayesian statistics, 
graphical Markov models, information retrieval, and Statistics education. He is also an Assistant Member at the Fred Hutchinson Cancer Research Center, and a Member at the National Center for Environmental Statistics, both in Seattle.

Daryl Pregibon is Head of the Statistics Research Group at AT\&T Labs. He received his Ph.D. degree in Statistics from the University of Toronto in 1979. His research interests include statistical modeling and computing especially as they relate to the analysis of very large data sets.

Padhraic Smyth received a Bachelor of Engineering degree from University College Galway, National University of Ireland in 1984 and an MSc and PhD in Electrical Engineering from the California Institute of Technology in 1985 and 1988 respectively. From 1988 to 1996 he was a member of technical staff at the Jet Propulsion Laboratory and he retains a visiting research position there. He is currently an assistant professor in the Information and Computer Science Department at the University of California, Irvine. His research interests span the interface between probability and computer science. 